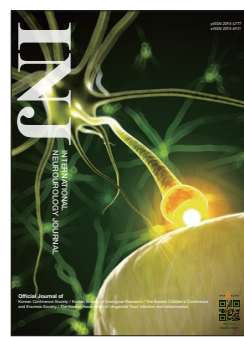

\title{
Current Understanding and Future Perspectives of Interstitial Cystitis/Bladder Pain Syndrome
}

\author{
Tomohiro Ueda ${ }^{1}$, Philip M. Hanno ${ }^{2}$, Ryoichi Saito ${ }^{1,3}$, Jane M. Meijlink ${ }^{4}$, Naoki Yoshimura ${ }^{1,5}$ \\ ${ }^{1}$ Department of Urology, Ueda Clinic \& Comfortable Urology Network, Kyoto, Japan \\ ${ }^{2}$ Department of Urology, Stanford University School of Medicine, Stanford, CA, USA \\ ${ }^{3}$ Department of Urology and Andrology, Kansai Medical University, Osaka, Japan \\ ${ }^{4}$ International Painful Bladder Foundation, Naarden, The Netherlands \\ ${ }^{5}$ Department of Urology, University of Pittsburgh School of Medicine, Pittsburgh, PA, USA
}

\begin{abstract}
Interstitial cystitis/bladder pain syndrome (IC/BPS) is a chronic disease characterized by suprapubic pain and lower urinary tract symptoms. Perhaps because of the heterogeneous nature of this disease and its multifactorial etiology, clinical trials in allinclusive populations of IC/BPS patients without phenotyping in the last decade have mainly failed to discover new therapeutic modalities of IC/BPS. Thus, phenotyping IC/BPS, aimed at identifying bladder-centric and/or bladder-beyond pathologies, including cystoscopic observation of Hunner or non-Hunner lesions of the bladder mucosa, is particularly important for the future of IC/BPS management. Based on recent discussions at international conferences, including the International Consultation on IC, Japan, it has been proposed that Hunner-lesion IC should be separated from other non-Hunner IC/BPS because of its distinct inflammatory profiles and epithelial denudation compared with non-Hunner IC/BPS. However, there are still no standard criteria for the diagnosis of Hunner lesions other than typical lesions, while conventional cystoscopic observations may miss atypical or small Hunner lesions. Furthermore, diagnosis of the bladder-centric phenotype of IC/BPS requires confirmation that identified mucosal lesions are truly a cause of bladder pain in IC/BPS patients. This review article discusses the current status of IC/BPS pathophysiology and diagnosis, as well as future directions of the proper diagnosis of bladder-centric IC/BPS, in which pathophysiological mechanisms other than those in inflammatory pathways, such as angiogenic and immunogenic abnormalities, could also be involved in both Hunner-lesion IC and non-Hunner IC/BPS. It is hoped that this new paradigm in the pathophysiological evaluation and diagnosis of IC/BPS could lead to pathology-based phenotyping and new treatments for this heterogeneous disease.
\end{abstract}

Keywords: Interstitial cystitis; Bladder pain syndrome; Hunner lesion; Cystoscopy; Narrow-band imaging; Angiogenesis

- Conflict of Interest: T. Ueda reports personal fees from Kyorin, Seikagaku and Japan Tobacco. P.M. Hanno has reports personal fees from Seikagaku, Ironwood, Imbrium, and HTL Biotech. N. Yoshimura reports personal fees from Kyorin and Astellas. R. Saito and J.M. Meijlink report no competing interests.

\section{INTRODUCTION}

Bladder pain syndrome (BPS) is an umbrella term encompassing bladder pain conditions, which include interstitial cystitis
(IC) with Hunner lesions, commonly seen in women and frequently confused with chronic pelvic pain syndrome/nonbacterial chronic prostatitis in men. BPS also often overlaps with irritable bowel syndrome, which occurs mainly in the large intes-

Corresponding author: Tomohiro Ueda (iD https://orcid.org/0000-0002-9611-5691 Department of Urology, Ueda Clinic, 6F CUBEOIKE, 599 Bano-cho, Anekojisagaru, Karasuma-dori, Nakagyo-ku, Kyoto 604-8172, Japan

Email: tom328177@gmail.com

Submitted: March 9, 2021 / Accepted after revision: April 13, 2021 
tine, and endometriosis, which may involve not only the uterus, but also the ovaries and bladder in women. Although there is still no globally accepted definition of IC/BPS, clinical trials in all-inclusive populations of IC/BPS patients without phenotyping in the last decade have mainly been unsuccessful. Thus, phenotyping IC/BPS, aimed at identifying bladder-centric and/ or bladder-beyond pathologies responsible for bladder pain and associated symptoms, is of paramount importance for the development of effective treatment modalities for IC/BPS. This review article will summarize current knowledge and future perspectives of IC/BPS diagnosis and treatment targets, with a particular focus on identifying bladder-centric IC/BPS pathophysiology.

\section{HISTORICAL AND CURRENT UNDERSTANDING OF IC/BPS: THE FUTURE (PRESENT) SEEN FROM THE PAST}

\section{Historical View of IC/BPS}

IC was initially reported as "a rare type of bladder ulcer in women" by Hunner [1] with characteristic changes in the bladder wall, which are known today as Hunner lesions [2] and associated with typical symptoms such as bladder pain, urinary frequency, and an urgent need to void [1,3-5]. In 1988, the National Institute of Diabetes and Digestive Kidney Diseases (NIDDK) developed a set of research criteria for IC. However, these criteria proved to be too strict for widespread clinical use [3]. Therefore, a more inclusive, symptom-based definition was developed and published as a guideline by the American Urological Association (AUA) in 2011 [3]. IC/BPS is defined in the AUA guideline as "an unpleasant sensation (pain, pressure and/ or discomfort) associated with lower urinary tract symptoms of more than 6-week duration, in the absence of infection or other identifiable causes" [3]. Cystoscopic findings are not a part of the definition, which is a purely symptom-based diagnosis of exclusion. In contrast, an East Asian guideline [6] divided IC/ BPS into 3 groups: (1) Hunner IC (HIC)/BPS with Hunner lesions, (2) non-Hunner IC /BPS with mucosal bleeding after hydrodistension in the absence of Hunner lesions, and (3) hypersensitive bladder (i.e., BPS) with no proven bladder pathology or other confusable disease. These categories are consistent with BPS type 3, type 2, and type 1, respectively, in the classification of the International Society for the Study of Bladder Pain Syndrome [7].

It has recently been reported that non-Hunner IC/BPS and
HIC are distinct pathological entities, with the latter being characterized by significant lymphocytic infiltration ( $\geq 200 \mathrm{cells} / \mathrm{mm}^{2}$ ) and epithelial denudation compared with non-Hunner IC/BPS patients [8]. A Hunner lesion seen in HIC patients is not an "ulcer," but rather a distinctive inflammatory lesion presenting as a reddened mucosal area with fragile microvessels radiating towards a central scar [9]. However, it has been demonstrated that mucosal bleeding after hydrodistension (i.e., glomerulations) seen in non-Hunner IC/BPS patients does not correlate with symptoms and is also found in patients without IC/BPS [10]. In this regard, the recently revised East Asian guideline categorized IC/BPS into 2 main entities: Hunner-type IC and nonHunner BPS [11]. However, bladder angiogenesis seen in nonHunner IC and HIC patients has also been recognized as an important characteristic in other chronic inflammatory diseases such as diabetic retinopathy, atherosclerosis, and inflammatory bowel disease $[12,13]$. Thus, although glomerulations shown by mucosal bleeding upon bladder hydrodistension are not unique to IC/BPS, underlying microvascular changes in the bladder mucosa due to angiogenesis could be another important component of the pathophysiology of both non-Hunner IC and HIC in addition to significant inflammation in the HIC subtype, and could potentially demonstrate a clinically significant phenotypic differentiation between non-Hunner IC and BPS without apparent bladder pathologies based on future research findings.

\section{Personal Experiences of IC/BPS}

More than a quarter of a century has passed since one of the authors (TU) became deeply involved in the clinical care of patients with IC/BPS, particularly IC with cystoscopically identified bladder pathologies, including Hunner lesions. It was while working at a Cancer Institute hospital in Tokyo, Japan in 1990 and encountering patients suffering from bladder pain similar to that of IC as an adverse reaction to intravesical bacillus Calmette-Guerin immunotherapy for bladder carcinoma in situ (CIS) that he was first motivated to become involved in IC/BPS. Some CIS patients who took the oral antiallergic agent suplatast tosilate (IPD) inexplicably experienced relief from their bladder pain. Later, after transferring to a local, general core hospital near Kyoto, Japan, he had a patient with refractory IC/BPS and severe bladder pain. Although she appeared to be a completely healthy, middle-aged woman, she said, "I want my bladder taken out." A cystoscopic examination revealed typical Hunner lesions (Fig. 1), and oral IPD therapy led to symptomatic relief. Experiences with 4 patients treated with IPD were subsequently 


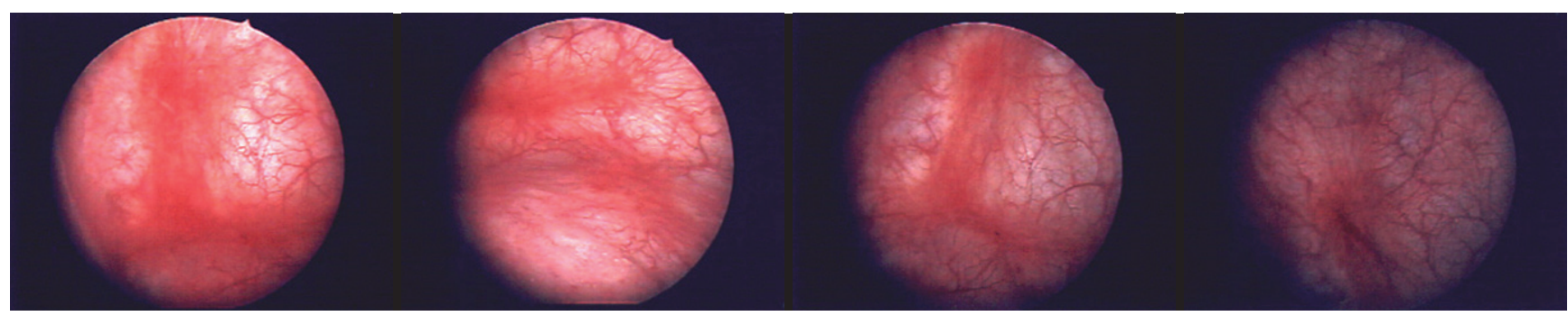

Fig. 1. Cystoscopic images of Hunner lesions. Typical Hunner lesions, based on the definition in the ESSIC classification (BPS type 3), in 4 different IC/BPS patients are shown. ESSIC, International Society for the Study of Bladder Pain Syndrome; BPS, bladder pain syndrome; IC, interstitial cystitis.

Table 1. Major outcomes of the International Consultation on Interstitial Cystitis, Japan (ICICIJ)

\begin{tabular}{|c|c|}
\hline ICICIJ & Major outcomes \\
\hline ICICJ \#1 (2003) & $\begin{array}{l}175 \text { Participants from } 17 \text { countries attended the first international meeting, which was a global kick off meeting for interstitial } \\
\text { cystitis with both doctors and patients }\end{array}$ \\
\hline \multirow[t]{2}{*}{ ICICJ \#2 (2007) } & 200 participants from 25 counties \\
\hline & - Definition of IC: bladder pain syndrome \& BPS/ IC \\
\hline \multirow[t]{2}{*}{ ICICJ \#3 (2013) } & 96 Participants from 16 countries \\
\hline & - Phenotyping of IC based on pathophysiology \\
\hline \multirow[t]{3}{*}{ ICICJ \#4 (2018) } & - Discussion on the definition of IC as bladder pain syndrome with Hunner lesion \\
\hline & . 179 Participants from 20 countries \\
\hline & $\begin{array}{l}\text { Consensus that Hunner lesion IC/BPS with significant inflammation in the bladder can be categorized as a separate disease } \\
\text { entity from other BPS conditions }\end{array}$ \\
\hline
\end{tabular}

BPS/IC, bladder pain syndrome/interstitial cystitis.

presented as a report on the clinical effects of IPD at the 1998 annual meeting of the AUA, and the results of IPD treatment were published in the Journal of Urology in 2000 [14]. Furthermore, this presentation at the 1998 AUA meeting offered a unique opportunity to make the acquaintance of key opinion leaders in the field of IC/BPS in the US, who encouraged the organization of international meetings with world experts in IC/BPS with the aim of achieving consensus on the definition and treatment of IC/BPS.

\section{Achievements Through the Meetings of the International Consultation on Interstitial Cystitis, Japan}

In 2003, the first meeting of International Consultation of Interstitial Cystitis, Japan (ICICJ) was successfully held and attended by 175 IC scientists from all over the world as well as a number of patient advocates. The objectives of the meeting included: (1) drawing public attention to IC/BPS, (2) allowing opinion exchanges among experts, and (3) presenting new findings. The conference was subsequently held again in 2007,

\section{3, and 2018 (Table 1) [15-18].}

During the meetings, we witnessed much discussion and debate regarding the differences in the definition, diagnosis, and treatment of IC/BPS in one and the same patient entirely depending on the healthcare system and culture of the countries/ regions where the participants resided. For example, in Asia and Europe, IC/BPS was diagnosed by performing cystoscopy and trying to define IC based on Hunner lesions and other bladder findings as a starting point, whereas in North America, IC/BPS was diagnosed as part of a symptom-based syndrome without a mandatory cystoscopic examination. Fifteen years ago, a research network program was established in the US known as the Multidisciplinary Approach to the Study of Chronic Pelvic Pain (MAPP) set up by the NIDDK. In this program, attempts have been made to classify IC/BPS through diagnostic methods other than cystoscopy or histological findings of the bladder alone, including urinary markers and brain imaging techniques. In addition to the MAPP efforts, several pharmaceutical companies have sponsored clinical trials for 


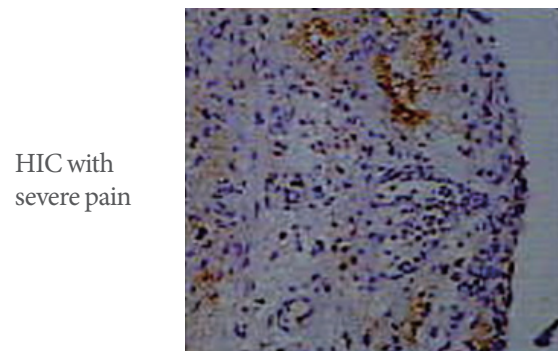

CD44

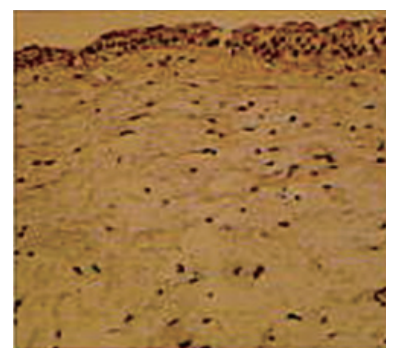

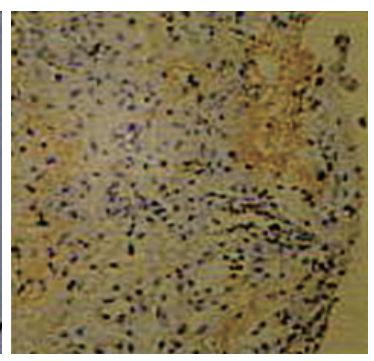

TGF- $\beta$

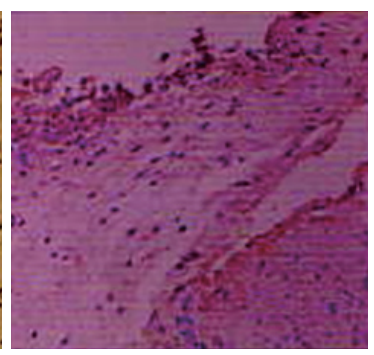

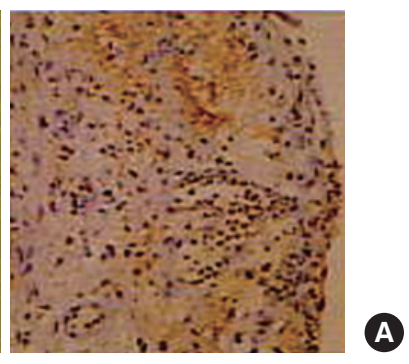

PD-ECGF/TP

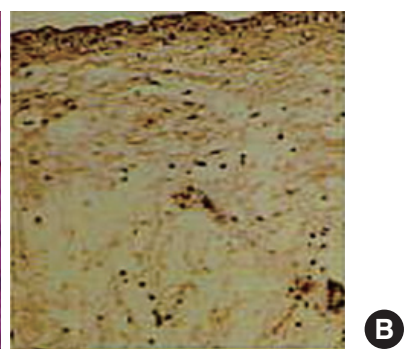

Fig. 2. Overexpression of angiogenic factors in the bladder of IC/BPS patients. The images show the results of immunohistochemical staining for epithelial and submucosal CD44, transforming growth factor (TGF)- $\beta$, and PD-ECGF/TP in bladder tissue sections from patients with IC/BPS. (A) Positive staining in the severe IC type (Hunner-type IC [HIC] patients with severe pain) is seen in the deeper part of the submucosal layer. (B) Positive staining in the mild IC type (non-Hunner-type patients with relatively mild pain) is present along the basement membrane of the bladder epithelium; $\times 200$ magnification. (Reproduced from Ueda T, et al. J Urol 2002;167:347-51 [32]). PD-ECGF, platelet-derived endothelial cell growth factor; TP, thymidine phosphorylase; IC, interstitial cystitis; BPS, bladder pain syndrome.

new investigational drugs in IC/BPS patients diagnosed primarily on the basis of symptoms alone in accordance with the current U.S. Food and Drug Administration guidance [19]. However, none of these trials have led to new approved treatments in the last decade. These outcomes highlight the importance of IC/BPS phenotyping, especially the differentiation of IC/BPS patients with cystoscopically identified Hunner lesions from non-Hunner IC/BPS, prompting reconsideration of the diagnostic approach [20]. Active discussions on this topic were also held at the ICICJ meetings, and the 2018 conference finally reached a consensus that HIC with significant inflammation in the bladder is clinically and pathologically distinct from nonHunner IC/BPS and may be categorized as a separate disease entity called "Hunner lesion disease (HLD)," distinct from other BPS conditions $[18,20]$. The importance of IC/BPS phenotyping using cystoscopy-based detection of Hunner lesions has been further supported by recent articles, including some from North America [20-23].

In addition, IPD was the first drug investigated in a clinical trial as drug therapy for IC/BPS in Japan. While this IPD clinical trial unfortunately did not meet the approval criteria set by the national health insurance coverage in Japan, it nevertheless revealed the potential implication of immune mechanisms and the important role of angiogenic factors in the pathogenesis of IC/BPS [14] (Fig. 2). Furthermore, the use of hydrodistension for the diagnosis of IC/BPS is known to have therapeutic benefits [24], which may shed light on the underlying pathogenesis of glomerulations after hydrodistension (Fig. 3). In other words, there are newly developed blood microvessels in the submucosa that are specific to IC/BPS and differ from normal blood vessels. Therefore, hydrodistension-induced stretching of the bladder wall could cause these microvessels to easily rupture and bleed [25] (Fig. 3).

However, a recent review of studies concluded that glomerulations caused by bladder hydrodistension are not a phenomenon unique to IC/BPS [10]. Similarly, the potassium chloride (KCL) test (potassium sensitivity test) [26] proposed by Parsons was also assessed as having low sensitivity and low specificity [27]. However, to date, underlying bladder conditions that might explain why glomerulations occur after hydrodistension and why pain is not triggered by potassium in some IC/BPS patients have not been fully clarified. Thus, we hypothesize that angiogenesis with microvessel formation in the bladder submucosa could be another important pathophysiological compo- 


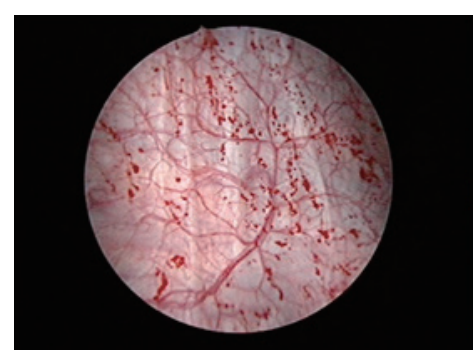

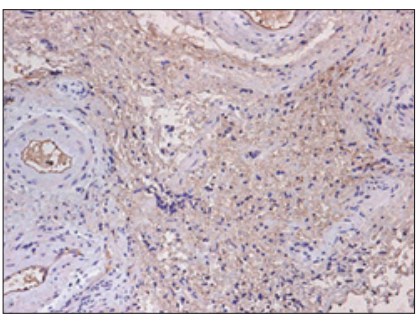

PD-ECGF* $(97.4 \%)$

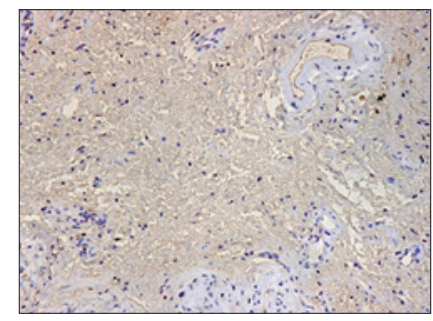

$\operatorname{VEGF}^{* *}(68.4 \%)$

$\left({ }^{*}{ }^{* *}\right.$ Negative staining in 5 control cases $)$

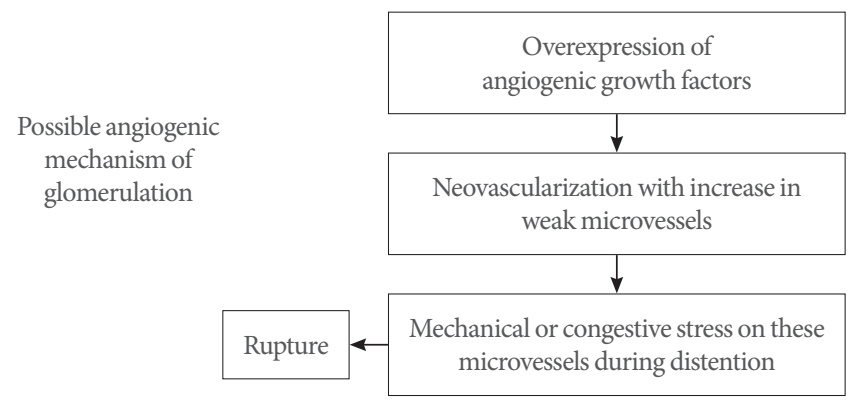

Fig. 3. The angiogenic mechanisms inducing glomerulations. In IC/BPS bladders, overexpression of angiogenic factors such as VEGF and PD-ECGF includes neovascularization with increases in weak microvessels, leading to rupture of microvessels during bladder distention, seen as glomerulations during cystoscopy (Reproduced from Ueda T, et al. J Urol 2002;167:347-51 [32]). IC, interstitial cystitis; BPS, bladder pain syndrome; VEGF, vascular endothelial growth factor; PD-ECGF, platelet-derived endothelial cell growth factor.

nent in the bladder of HIC and non-Hunner IC/BPS, in addition to inflammatory changes in HIC bladders, despite the fact that glomerulation upon hydrodistension is not in itself a phenomenon specific to IC/BPS bladders. Fig. 4 summarizes the potential immunogenic mechanisms underlying angiogenic changes in the bladder submucosa of bladder-centric IC/BPS. Firstly, as part of multiple etiologies, disturbances in the glycosaminoglycan (GAG) layer with increased bladder epithelial permeability lead to activation of inflammatory responses and overexpression of angiogenic factors, such as vascular endothelial growth factor (VEGF) and platelet-derived endothelial cell growth factor (PD-ECGF) in the submucosa. Furthermore, disruption of the GAG layer causes the release of adhesion factors such as CD44 and transforming growth factor-beta, which are known to bind with angiogenic factors that are present in very small amounts via heparin-binding and become solidified. This process has been elucidated as part of the wound healing process; thus, it is considered that the overexpression of angiogenic factors is involved in tissue fibrosis leading to bladder atrophy [25]. Submucosal inflammatory cell infiltration with cytokine/chemokine production, as well as increased bladder epithelial permeability allowing harmful urinary substances such as $\mathrm{K}^{+}$and $\mathrm{H}^{+}$ions to penetrate the bladder wall, is also presumed to sensitize bladder afferent pathways, especially the Cfiber type, thereby causing IC/BPS symptoms such as pain and urgency (Fig. 4).

\section{FUTURE PERSPECTIVES WITH OUR PROPOSALS}

\section{Current Problems and Controversies Regarding IC/BPS Collectively assuming that HLD and non-Hunner IC/BPS is one disease entity has become a major impediment to identifying factors responsible for bladder pain}

As mentioned above, IC/BPS is part of the pelvic pain spectrum of disorders that can be caused by impairments in the urinary, gynecologic, and gastrointestinal systems; therefore, evaluation of each organ is necessary. In addition, in patients diagnosed with chronic prostatitis, IC/BPS has been reported as a comorbidity associated with decreases in maximal voided volume per micturition and maximal bladder capacity during cystoscopy under local anesthesia. Therefore, a cystoscopic diagnosis may be helpful for the differential diagnosis of chronic prostatitis and associated comorbidities [28]. 


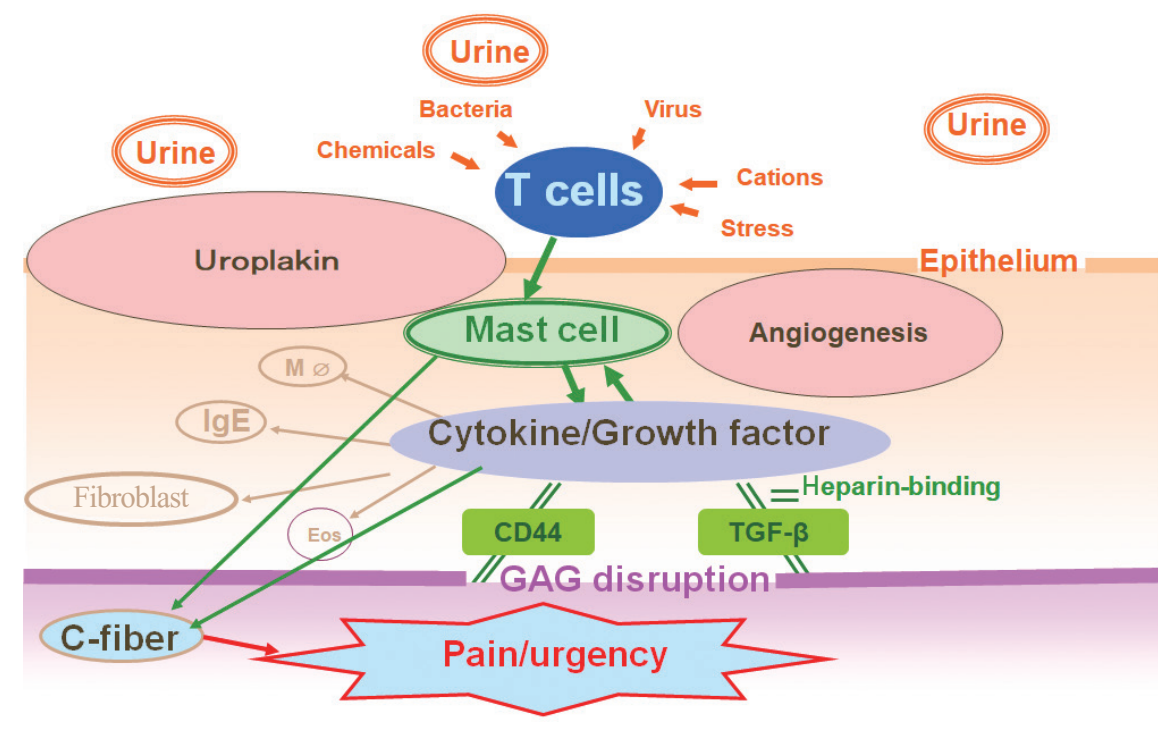

Fig. 4. Immunogenic and angiogenic pathophysiology of IC/BPS. In bladder-centric IC/BPS, increased bladder epithelial permeability due to disturbances in the glycosaminoglycan (GAG) layer and activation of inflammatory cells including mast cells, T cells, and B cells has been proposed as part of multiple etiologies. Disturbance of the bladder epithelium, overexpression of angiogenic factors, such as platelet-derived endothelial cell growth factor (PD-ECGF), occurs in the submucosa. These angiogenic factors are present in very small amounts in the body or bladder under normal conditions. However, angiogenic factor overexpression in IC/BPS may result from abnormalities in GAG because disruption of the GAG layer, which is a protective factor of the bladder epithelium, causes the release of adhesion factors in the GAG layer, such as CD44 and TGF- $\beta$ (an adhesion molecule), which are known to bind with angiogenic factors that are present in very small amounts via heparin-binding and become solidified. This has been elucidated as part of the wound healing process; thus, it is considered that overexpression of angiogenic factors is involved in tissue fibrosis leading to bladder atrophy. The onset of IC/BPS starts with various insults to the bladder epithelium, for example by previous bacterial infection and/or harmful substances such as $\mathrm{K}^{+}$and $\mathrm{H}^{+}$ions in the urine. These noxious stimuli are detected by inflammatory cells such as type 2 helper T lymphocytes, which then send signals to activate mast cells. Mast cells play an important role in the inflammatory process to induce angiogenic factors and immunostimulatory factors to activate other immunocompetent cells. These stimulating factors are presumed to sensitize bladder afferent pathways, especially the C-fiber type, thereby causing IC/BPS symptoms (pain and urgency). M $\Phi$, macrophage; Eos, eosinophils; IC, interstitial cystitis; BPS, bladder pain syndrome; TGF, transforming growth factor.

\section{Bladder pain related to the nervous, endocrine, and immune systems}

The International Continence Society meeting held in Korea in 2002 defined the term "overactive bladder (OAB) syndrome" as a frequency-urgency syndrome with a chief complaint of sudden urinary urgency that is likely to show uninhibited bladder contractions (i.e., detrusor overactivity) if bladder pressure is measured by cystometry. This is where the concept of $\mathrm{OAB}$ originated. However, the frequency-urgency syndrome has previously been used to refer to IC [29] (Fig. 5), suggesting a symptomatic overlap between IC and OAB. Neuromodulation using Interstim implantation is reimbursed in the United States for IC/BPS not because it has been approved for this indication, but because IC/BPS can be identified as part of the frequency/ urgency syndrome, which is used to refer to OAB. Recent stud- ies have also indicated considerable overlap between $\mathrm{OAB}$ and IC/BPS symptoms [30]. Therefore, it is necessary to evaluate whether bladder pain is related to other etiologies, including $\mathrm{OAB}$ induced by lower urinary tract dysfunction, endocrinological abnormalities due to gynecological diseases such as endometriosis, and immunological abnormalities such as inflammatory bowel disease.

\section{Relationship with refractory $O A B$}

Although IC/BPS is a diagnosis of exclusion with regard to $\mathrm{OAB}$, it has not been specified how $\mathrm{OAB}$ should be excluded. Therefore, in refractory $\mathrm{OAB}$, it can be useful to proactively perform a cystoscopic examination to confirm the presence or absence of intravesical lesions including Hunner lesions. 


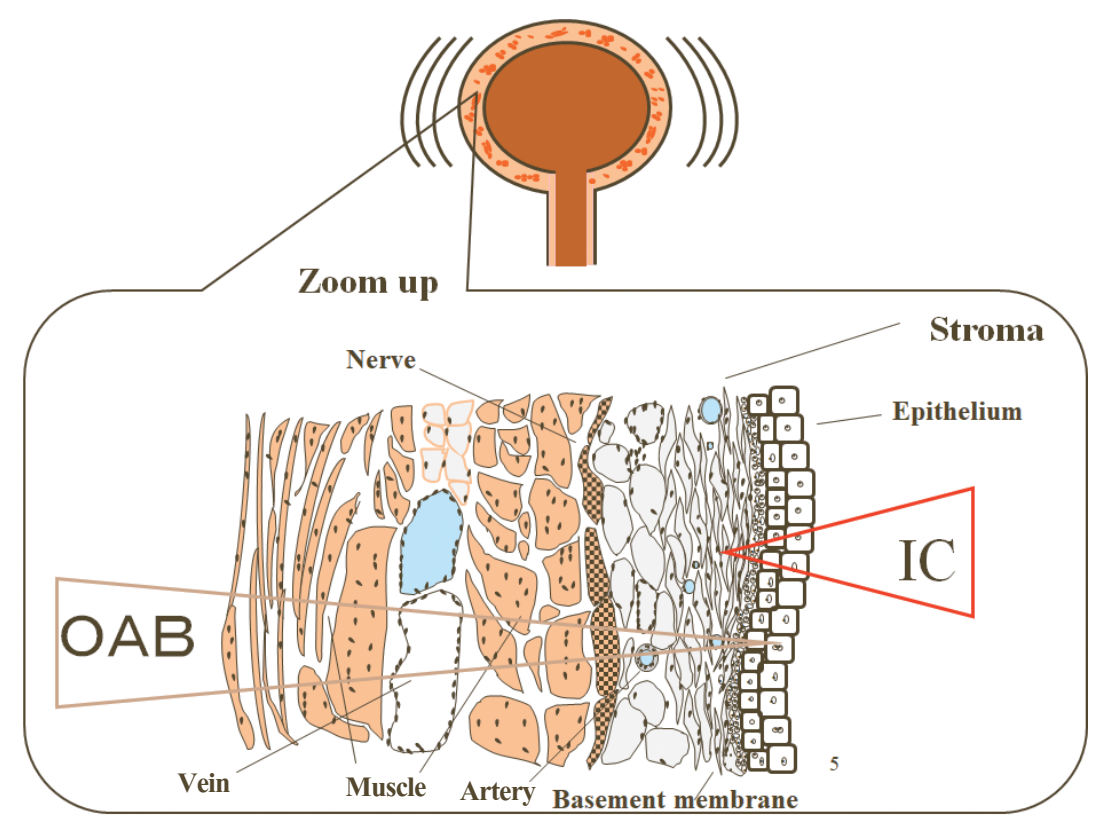

Fig. 5. Symptomatic and pathophysiological overlaps between IC and OAB as the frequency-urgency syndrome. Bladder-centric IC and $\mathrm{OAB}$ exhibit pathophysiological changes at a higher degree in the epithelial/submucosal and submucosal/muscle regions, respectively; however, hallmark symptoms such as urgency are often found in both disease conditions. IC, interstitial cystitis; OAB, overactive bladder.

\section{Concepts for classification of pain etiologies, either bladder-centric or bladder-beyond, or both in IC/BPS}

Finally, it is important to evaluate whether bladder pain is caused by bladder lesions or attributable to extrabladder pathophysiology. It should also be noted that bladder-centric and bladder-beyond etiologies may coexist to induce the overall symptoms in IC/BPS.

\section{Future Directions and Proposals}

As described above, in order to establish a diagnosis of bladdercentric IC/BPS in patients complaining of bladder pain, the diagnostic criterion is to confirm the presence of a lesion in the bladder mucosa by cystoscopy. However, there are problems concerning the accuracy of cystoscopic diagnosis (Table 2). A review paper presented at the 2018 ICICJ meeting reported that the proportion of Hunner lesions in IC/BPS patients with bladder pain varied greatly among centers, ranging from $5 \%$ to $57 \%$ [31].

\section{The challenge concerning the accuracy of cystoscopic findings is to identify what can be used as "true" indicators (Table 2)}

With regard to glomerulations caused by hydrodistension, a consensus has been reached that this phenomenon is not specific to IC/BPS [10]. However, it has previously been reported that overstretching the bladder wall during bladder filling may
Table 2. Issues on validation of cystoscopic findings in terms of accuracy, precision, sensitivity and specificity

\begin{tabular}{ll}
\hline Parameter & \multicolumn{1}{c}{ Issues } \\
\hline Accuracy & - What is true? \\
& - Glomerulation?, inflammatory cell?, angiogenic factor?, \\
& $\begin{array}{l}\text { angiogenesis with neovasculization? } \\
\text { - What is Hunner lesion? }\end{array}$ \\
Precision & - Grading \\
& - Decision of severity \\
Sensitivity & - Differentiation; CIS or OAB \\
Specificity & - What is normal? \\
& - Threshold setting
\end{tabular}

CIS, carcinoma in situ; $\mathrm{OAB}$, overactive bladder.

be responsible for the rupture of newly developed blood microvessels in non-Hunner lesion-containing bladders of IC/BPS patients [25] and that overexpression of PD-ECGF or VEGF in the bladder was found in both non-Hunner IC and HIC subtypes with higher expression in the latter than in the former [32]. This means that the underlying condition for neovascularization, particularly involving the overexpression of angiogenic factors such as PD-ECGF or VEGF, should be considered to be bladder lesions rather than glomerulations (Fig. 4) [25,32]. 
With respect to Hunner lesions, a pathological mechanism involving specific inflammatory cell infiltration has been elucidated [33] and reportedly accompanied by the overexpression of angiogenic factors [34]. Therefore, further investigation is needed regarding the angiogenesis-related pathophysiology of bladder-centric HIC and non-Hunner IC. Additionally, we have reported that the use of narrow-band imaging (NBI) enables sensitive detection of angiogenesis and neovascularization in the bladder mucosa during cystoscopy, making it possible to accurately identify lesions in the bladder mucosa accompanied by Hunner lesions and new blood microvessel formation (Fig. 6) [35].

Furthermore, we have recently completed a successful, multicenter, randomized, double-blind, placebo-controlled clinical trial of intravesical KRP-116D, 50\% dimethyl sulfoxide (DMSO) solution in IC/BPS patients in Japan [36]. In this trial, we recruited IC/BPS patients with the bladder-centric phenotype, which was confirmed by the existence of bladder pathologies such as Hunner lesion and/or mucosal bleeding during hydrodistension (i.e., glomerulations). Also, because glomerulations may not be IC/BPS-specific or responsible for bladder symptoms [10], all study participants were confirmed to exhibit short-term bladder pain relief following intravesical lidocaine instillation during the placebo run-in period, verifying that their symptoms originated in the bladder (i.e., bladder-centric phenotype). For the primary endpoint, the change in the mean O'Leary-Sant interstitial cystitis symptom index score was significantly greater in the KRP-116D group than in the placebo group. Statistically significant improvements for KRP-116D were also observed in the secondary endpoints, including the O'Leary-Sant interstitial cystitis problem index, micturition episodes per 24 hours, voided volume per micturition, maximum voided volume per micturition, numerical rating scale for bladder pain, and global response assessment. Thus, this successful DMSO clinical trial indicates that careful phenotyping and selection of patients suited to the clinical trial objectives seems particularly important for the success of clinical trials in IC/BPS patients who have heterogeneous pathological backgrounds.

\section{The precision of cystoscopic findings}

The challenge lies in establishing a diagnostic accuracy scale, such as grading of bladder lesions and a severity assessment method (Table 2).

\section{Another issue concerning the sensitivity of cystoscopic findings is diagnostic differentiation from bladder tumors}

Where Hunner lesions are concerned, the concurrent use of NBI can improve detection sensitivity [35]; however, distin-

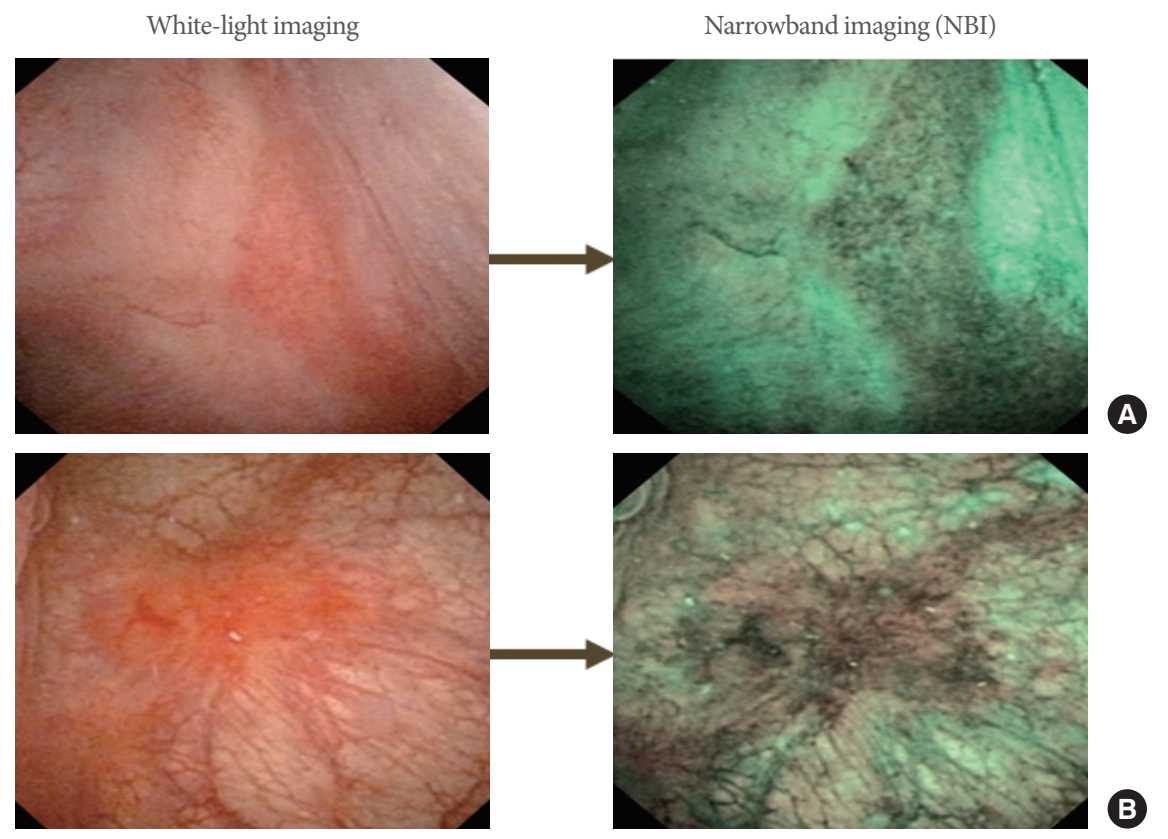

Fig. 6. Hunner lesions: white-light imaging (A) and narrow-band imaging (B) in 2 patients with IC/BPS. IC, interstitial cystitis; BPS, bladder pain syndrome. 
guishing Hunner lesions from bladder CIS remains a challenge. Without a histological examination, the 2 conditions cannot be differentiated. In cases of $\mathrm{OAB}$, treatment is usually decided on the basis of symptoms without confirming bladder lesions in patients complaining of urinary urgency, who are likely to show uninhibited bladder contractions in urodynamic studies. Therefore, depending on the severity of bladder pain, OAB is very likely to overlap with IC/BPS (Fig. 5). Particularly in cases of refractory $\mathrm{OAB}$, as well as patients who report urgency and bladder pain, pressure, or discomfort associated with filling, the differential diagnosis of IC/BPS is aided by a determination of bladder pathology with the aid of cystoscopy and with urodynamic evaluations.

\section{The specificity of cystoscopic findings}

As the first step, the criteria for defining the normal bladder are important. Thus, knowing the histo-anatomical structure and endoscopic appearance of the normal bladder mucosa is essential. However, unlike gastroenterological screening tests such as gastroscopy or colonoscopy, we consider that urologists, unfortunately, have very few opportunities to see normal cystoscopic findings, since cystoscopy is most often performed in patients who already exhibit some kinds of lower urinary tract symptoms. Fig. 7 depicts representative cystoscopic images of normal and non-Hunner IC bladders, which respectively show the whitish, distensible mucosa with multiple mucosal folds in the former (Fig. 7A), and the stretched, hypervascular mucosa without mucosal folding in the latter (Fig. 7B). Moreover, in NBI-assisted cystometry, hypervascularity in the reddish bladder mucosa is more easily recognized in non-Hunner IC (Fig. 7C). Therefore, it is potentially a problem that most urologists see the bladder epithelium when it is no longer in a normal state (i.e., abnormal). Therefore, the bladder mucosa that urologists consider to be normal may often, in fact, already be abnormal, as shown in Fig. 7B and C, possibly causing a delay in diagnosing bladder lesions associated with IC/BPS. In addition, abnormal morphology of the bladder epithelium does not necessarily mean that it is pathological. It should only be considered to be pathological when an abnormal epithelium is found in association with bladder pain and other symptoms. Therefore, it is important for the definition of bladder-centric IC/BPS to accurately identify bladder pathologies such as Hunner lesions or neovascular changes on the bladder mucosal surface and, at the same time, to confirm that they are responsible for causing bladder pain. Thus, it is necessary to standardize the methodology of the cystoscopic assessment of Hunner lesions. A recent article by Ronstrom and Lai presented an informative atlas showing cystoscopic photos of typical Hunner lesions in IC/BPS patients [37]. However, there is still a need to define atypical or small Hunner lesions, which will be missed under conventional cystoscopy with white light [35]. In addition, for the diagnosis of bladder-centric, non-Hunner IC/BPS, the detection of mucosal hypervascular lesions caused by angiogenesis and differing from normal mucosa (Fig. 7) needs to be standardized.

Furthermore, with regard to bladder-centric, non-Hunner IC/BPS, it is also important to identify whether bladder lesions are associated with urothelial hyperpermeability, which is induced by defective, non-self-repairing GAG layer and allows
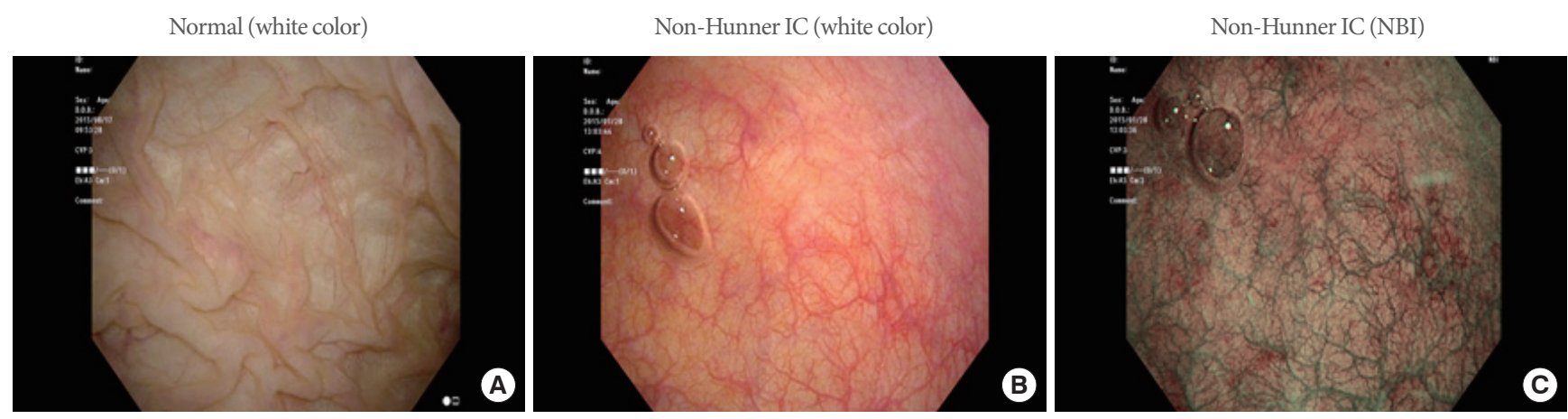

Fig. 7. Representative cystoscopic images of the normal bladder (A) and the non-Hunner IC bladder under white-light imaging (B) or narrow-band imaging (NBI) (C). (A) The bladder epithelial mucosa is largely whitish in color with multiple folds on the mucosal surface in the normal bladder. Furthermore, the normal mucosal epithelium is stretchable in all directions during bladder distention because it is loosely connected to the underlying muscle layer. (B, C) Abnormal bladder epithelium characterized by stretched, hypervascular mucosa without mucosal folds in the non-Hunner IC bladder, the appearance of which is similar to the so-called "lead pipe sign" of ulcerative colitis during colonoscopy. IC, interstitial cystitis. 
urinary substances to penetrate the bladder wall and induce bladder pain [5]. In this regard, noninvasive bladder imaging using magnetic resonance imaging has been investigated to evaluate an increase in the bladder mucosal permeability due to bladder mucosal lesions as a diagnostic method to replace the KCI sensitivity test, and expectations for the outcomes are high [38].

\section{Biomarkers: causes, results, or completely independent factors?}

Various biomarkers have been reported to diagnose IC/BPS and assess the therapeutic effects. Although there is no doubt about the changes in expression levels in the bladder or urine, it is necessary to clarify whether these changes result from the host's response to the disease (results), are induced by the disease (causes), or occur as completely independent factors (bystanders). For example, in the case of bladder cancer, the aforementioned excessive expression of angiogenic factors in the bladder mucosa is triggered by the invasion of malignant cells, whereas the change in angiogenic factors with neovascularization in IC/BPS is considered to be due to the promotion of healing in response to inflammation on the host side [32]. Thus, even in patients presenting with the same symptoms and showing equal overexpression of angiogenic factors as biomarkers, the underlying pathological mechanisms can be completely different. Therefore, if various biomarkers are detected, each needs to be investigated with a highly accurate method to determine whether it is a cause, a result, or just a bystander.

\section{Need for science-based dietary regimens}

Finally, although it is common knowledge that urine $\mathrm{pH}$ is influenced by diet and that acidic urine enhances bladder pain and frequent urination, diet modification therapy is often not vigorously enforced in IC/BPS patients [39]. Some studies have also reported that substances such as potassium and toxic cations in urine can modify lower urinary tract symptoms of IC/ BPS [40] and that various dietary factors including citrus fruits, tomatoes, spicy food, alcohol, and caffeinated beverages can trigger a flare (defined as urologic or pelvic pain that is much worse than usual) in IC/BPS patients [41]. Therefore, further research in this field of dietary modification is strongly recommended to improve the treatment of IC/BPS.

\section{CONCLUSION}

This review article has summarized the history of research findings on HIC and non-Hunner IC/BPS in the past quarter of a century and made some critical proposals for the future. Although great efforts have been made regarding the definition of this condition, its pathophysiology, and new treatments, various clinical studies performed in the last decade have unfortunately so far failed to lead to new treatments, which may indicate that there has been insufficient effort focused on exploring the etiology, pathogenesis, and phenotyping of this heterogeneous disease.

Therapeutic effects of treatments cannot be obtained unless we initially address the patients' symptoms and explore the cause. Despite the tremendous medical advances made by developing many testing modalities and biomarkers, there can be no successful treatment in the absence of an accurate diagnosis. Thus, there can be no bright future for IC/BPS without these 3 steps: (1) understanding the symptoms, (2) detecting abnormal findings in or outside the bladder, and (3) verifying that the abnormality is the cause of the symptoms.

\section{ORCID}

$\begin{array}{ll}\text { Tomohiro Ueda } & 0000-0002-9611-5691 \\ \text { Philip M. Hanno } & 0000-0002-6597-9155 \\ \text { Ryoichi Saito } & 0000-0002-3163-8723 \\ \text { Jane M. Meijlink } & 0000-0002-0943-2390 \\ \text { Naoki Yoshimura } & 0000-0001-8070-1664\end{array}$

\section{AUTHOR CONTRIBUTION STATEMENT}

- Conceptualization: TU, PH, JM, NY

- Project administration: TU

-Writing-original draft: $T U, N Y$

- Writing-review \& editing: $T U, P H, R S, J M, N Y$

\section{REFERENCES}

1. Hunner G. A rare type of bladder ulcer in women; report of cases. Boston Med Surg J 1915;172:660-4.

2. Fall M, Logadottir Y, Peeker R. Interstitial cystitis is bladder pain syndrome with Hunner's lesion. Int J Urol 2014;21 Suppl 1:79-82.

3. Hanno PM, Burks DA, Clemens JQ, Dmochowski RR, Erickson D, Fitzgerald MP, et al. AUA guideline for the diagnosis and treat- 
ment of interstitial cystitis/bladder pain syndrome. J Urol 2011;185: 2162-70.

4. Homma Y, Ueda T, Ito T, Takei M, Tomoe H. Japanese guideline for diagnosis and treatment of interstitial cystitis. Int J Urol 2009; 16:4-16.

5. Ogawa T, Ishizuka O, Ueda T, Tyagi P, Chancellor MB, Yoshimura $\mathrm{N}$. Current and emerging drugs for interstitial cystitis/bladder pain syndrome (IC/BPS). Expert Opin Emerg Drugs 2015;20:555-70.

6. Homma Y, Ueda T, Tomoe H, Lin AT, Kuo HC, Lee MH, et al. Clinical guidelines for interstitial cystitis and hypersensitive bladder syndrome. Int J Urol 2009;16:597-615.

7. van de Merwe JP, Nordling J, Bouchelouche P, Bouchelouche K, Cervigni M, Daha LK, et al. Diagnostic criteria, classification, and nomenclature for painful bladder syndrome/interstitial cystitis: an ESSIC proposal. Eur Urol 2008;53:60-7.

8. Maeda D, Akiyama Y, Morikawa T, Kunita A, Ota Y, Katoh H, et al. Hunner-type (classic) interstitial cystitis: a distinct inflammatory disorder characterized by pancystitis, with frequent expansion of clonal b-cells and epithelial denudation. PLoS One 2015;10:e0143316.

9. Akiyama Y, Maeda D, Morikawa T, Niimi A, Nomiya A, Yamada Y, et al. Digital quantitative analysis of mast cell infiltration in interstitial cystitis. Neurourol Urodyn 2018;37:650-7.

10. Wennevik GE, Meijlink JM, Hanno P, Nordling J. The role of glomerulations in bladder pain syndrome: a review. J Urol 2016;195: 19-25.

11. Homma Y, Akiyama Y, Tomoe H, Furuta A, Ueda T, Maeda D, et al. Clinical guidelines for interstitial cystitis/bladder pain syndrome. Int J Urol 2020;27:578-89.

12. Carmeliet $P$, Jain RK. Angiogenesis in cancer and other diseases. Nature 2000;407:249-57.

13. Chidlow JH Jr, Shukla D, Grisham MB, Kevil CG. Pathogenic angiogenesis in IBD and experimental colitis: new ideas and therapeutic avenues. Am J Physiol Gastrointest Liver Physiol 2007;293: G5-18.

14. Ueda T, Tamaki M, Ogawa O, Yamauchi T, Yoshimura N. Improvement of interstitial cystitis symptoms and problems that developed during treatment with oral IPD-1151T. J Urol 2000;164:1917-20.

15. Ueda T. The legendary beginning of the International Consultation on Interstitial Cystitis. Int J Urol 2003;10 Suppl:S1-2.

16. Ueda T. What should we do for interstitial cystitis. In: 2nd International Consultation on Interstitial Cystitis Japan (ICICJ); $2007 \mathrm{Mar}$ 23-25; Kyoto, Japan.

17. Hanno PM. Preface. 3rd International Consultation on Interstitial Cystitis Japan (ICICJ) and International Society for the Study of Bladder Pain Syndrome (ESSIC) Joint Meeting, 21-23 March 2013,
Kyoto, Japan. Int J Urol 2014;21(Suppl 1):3.

18. Ueda T. Introduction of ICICJ 15 years. Int J Urol 2019;26 Suppl $1: 1-2$.

19. Nickel JC, Moldwin R. FDA BRUDAC 2018 criteria for interstitial cystitis/bladder pain syndrome clinical trials: future direction for research. J Urol 2018;200:39-42.

20. Fall M, Nordling J, Cervigni M, Dinis Oliveira P, Fariello J, Hanno P, et al. Hunner lesion disease differs in diagnosis, treatment and outcome from bladder pain syndrome: an ESSIC working group report. Scand J Urol 2020;54:91-8.

21. Nickel JC. Comments to Editorial by J. Curtis Nickel. It is premature to categorize Hunner lesion interstitial cystitis as a distinct disease entity. Scandinavian Journal of Urology 2020, Vol. 54, No. 2, 99-100. Scand J Urol 2020;54:357.

22. Hanno P, Fall M, Meijlink J, Nordling J. Towards a new paradigm in bladder pain syndrome and interstitial cystitis. BJU Int 2020; 126:549-50.

23. Doiron RC, Tolls V, Irvine-Bird K, Kelly KL, Nickel JC. Clinical phenotyping does not differentiate hunner lesion subtype of interstitial cystitis/bladder pain syndrome: a relook at the role of cystoscopy. J Urol 2016;196:1136-40.

24. Niimi A, Nomiya A, Yamada Y, Suzuki M, Fujimura T, Fukuhara H, et al. Hydrodistension with or without fulguration of hunner lesions for interstitial cystitis: long-term outcomes and prognostic predictors. Neurourol Urodyn 2016;35:965-9.

25. Tamaki M, Saito R, Ogawa O, Yoshimura N, Ueda T. Possible mechanisms inducing glomerulations in interstitial cystitis: relationship between endoscopic findings and expression of angiogenic growth factors. J Urol 2004;172:945-8.

26. Parsons CL, Zupkas P, Parsons JK. Intravesical potassium sensitivity in patients with interstitial cystitis and urethral syndrome. Urology 2001;57:428-32.

27. Hanno P. Potassium sensitivity test for painful bladder syndrome/ interstitial cystitis: con. J Urol 2009;182:431-2,434.

28. Ueda M, Sengiku A, Kono J, Negoro H, Saito R, Yoshimura N, et al. Low bladder capacity is an important predictor for comorbidity of interstitial cystitis with Hunner's lesion in patients with refractory chronic prostatitis/chronic pelvic pain syndrome. Int J Urol 2019; 26 Suppl 1:53-6.

29. Ueda T, Sant GR, Hanno PM, Yoshimura N. Interstitial cystitis and frequency-urgency syndrome (OAB syndrome). Int J Urol 2003;10 Suppl:S39-48.

30. Ackerman AL, Lai HH, Parameshwar PS, Eilber KS, Anger JT. Symptomatic overlap in overactive bladder and interstitial cystitis/ bladder pain syndrome: development of a new algorithm. BJU Int 
2019;123:682-93.

31. Whitmore KE, Fall M, Sengiku A, Tomoe H, Logadottir Y, Kim YH. Hunner lesion versus non-Hunner lesion interstitial cystitis/ bladder pain syndrome. Int J Urol 2019;26 Suppl 1:26-34.

32. Ueda T, Tamaki M, Ogawa O, Yoshimura N. Over expression of platelet-derived endothelial cell growth factor/thymidine phosphorylase in patients with interstitial cystitis and bladder carcinoma. J Urol 2002;167:347-51.

33. Akiyama Y, Maeda D, Katoh H, Morikawa T, Niimi A, Nomiya A, et al. Molecular taxonomy of interstitial cystitis/bladder pain syndrome based on whole transcriptome profiling by next-generation RNA sequencing of bladder mucosal biopsies. J Urol 2019;202:290300.

34. Furuta A, Suzuki Y, Igarashi T, Koike Y, Kimura T, Egawa S, et al. Angiogenesis in bladder tissues is strongly correlated with urinary frequency and bladder pain in patients with interstitial cystitis/ bladder pain syndrome. Int J Urol 2019;26 Suppl 1:35-40.

35. Ueda T, Nakagawa M, Okamura M, Tanoue H, Yoshida H, Yoshimura N. New cystoscopic diagnosis for interstitial cystitis/painful bladder syndrome using narrow-band imaging system. Int J Urol 2008;15:1039-43.

36. Yoshimura N, Homma Y, Tomoe H, Otsuka A, Kitta T, Masumori
$\mathrm{N}$, et al. Efficacy and safety of intravesical instillation of KRP-116D (50\% dimethyl sulfoxide solution) for interstitial cystitis/bladder pain syndrome in Japanese patients: a multicenter, randomized, double-blind, placebo-controlled, clinical study. Int J Urol 2021;28: 545-53.

37. Ronstrom C, Lai HH. Presenting an atlas of Hunner lesions in interstitial cystitis which can be identified with office cystoscopy. Neurourol Urodyn 2020;39:2394-400.

38. Tyagi P, Moon CH, Janicki J, Kaufman J, Chancellor M, Yoshimura $\mathrm{N}$, et al. Recent advances in imaging and understanding interstitial cystitis. F1000Res 2018;7:F1000 Faculty Rev-1771.

39. Ueda T, Yoshida T, Tanoue H, Ito M, Tamaki M, Ito Y, et al. Urine alkalization improves the problems of pain and sleep in hypersensitive bladder syndrome. Int J Urol 2014;21:512-7.

40. Parsons CL, Argade S, Evans RJ, Proctor J, Nickel JC, Rosenberg MT, et al. Role of urinary cations in the etiology of interstitial cystitis: a multisite study. Int J Urol 2020;27:731-5.

41. Lai HH, Vetter J, Song J, Andriole GL, Colditz GA, Sutcliffe S. Management of symptom flares and patient-reported flare triggers in interstitial cystitis/bladder pain syndrome (IC/BPS)-findings from one site of the MAPP research network. Urology 2019; 126:24-33. 